\title{
Tremor mimicking ventricular tachycardia
}

\author{
Pieter Martens MD, Peter Sinnaeve MD PhD
}

$\mathrm{A}$ 92-year-old woman was admitted to hospital with pneumonia. Her medical history included a percutaneous coronary intervention and subsequent diagnosis of ischemic cardiomyopathy 15 years earlier. While in hospital, epigastric and retrosternal pain associated with nausea developed in the patient. On physical examination, she was anxious and tremulous. Her blood pressure was $145 / 85 \mathrm{~mm} \mathrm{Hg}$, her pulse was regular at 75 beats/min, and she was afebrile. On cardiac auscultation, she had normal first and second heart sounds, a regular rhythm of 75 beats/min and no audible murmur. At first glance, simultaneous bedside analysis of an electrocardiographic (ECG) tracing suggested the presence of a broad QRS complex tachycardia (Figure 1). Because the patient was anxious, the on-call doctor held her left hand during the test. As a consequence, the patient's left hand was not shaking during the ECG tracing, unlike the right hand. Because lead III showed a normal sinus rhythm and the observed clinical picture did not suggest ventricular tachycardia, a diagnosis of tremor-induced artifact was proposed.

Tremor can cause ECG artifacts that mimic a broad QRS complex tachycardia. ${ }^{1}$ Characteristics allowing differentiation from a broad QRS complex tachycardia include the finding of normal QRS complexes between artifact waves (Figure 1, small arrows) or an unstable baseline at the beginning of the ECG recording (not shown). One of the upper extremities is often free of tremor, which results in a normal ECG tracing in one of the frontal leads (Figure 1, large arrow). ${ }^{2}$ A simple bedside evaluation allows for the recognition of important clinical clues, such as a tremulous patient without hemodynamic compromise and detection of a normal pulse during palpitation or cardiac auscultation. Misdiagnosing artifacts as ventricular tachycardia may result in patients having to undergo a range of unnecessary diagnostic and therapeutic procedures, including electrophysiologic testing, anti-

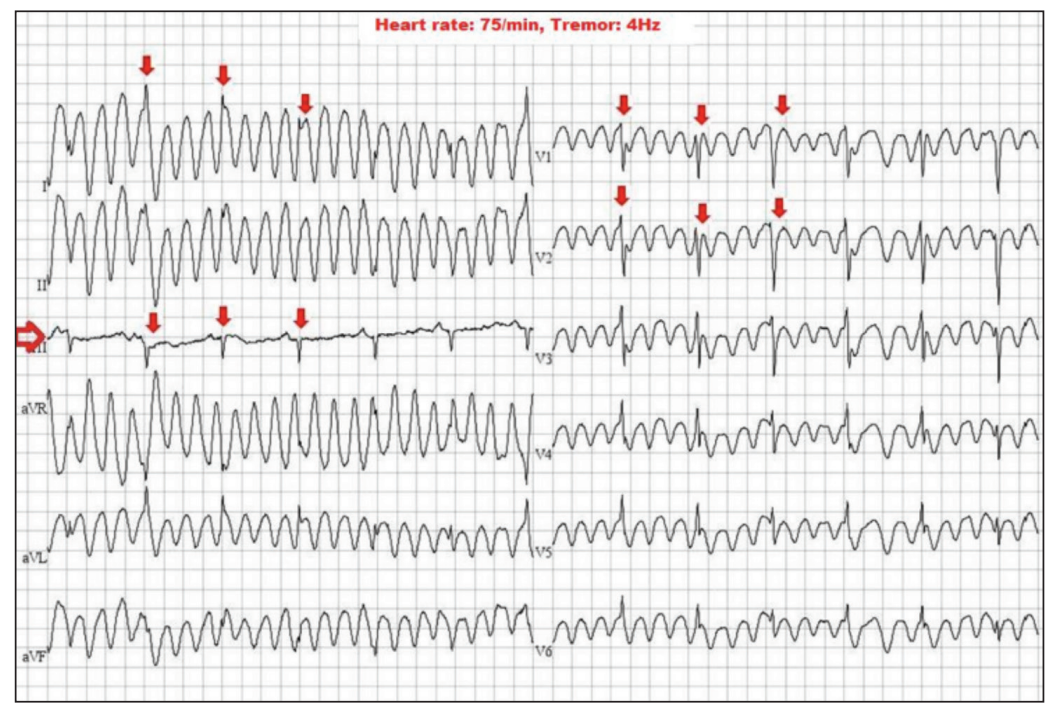

Figure 1: Electrocardiogram showing a tremor $(4 \mathrm{~Hz})$ causing an electrocardiographic artifact. Normal QRS complexes can be seen interspersed between artifact waves (small arrows). A normal sinus rhythm is seen in frontal lead III (large arrow). The amplitude of the registered tremor is more pronounced in the tracings from the frontal leads than in those from the precordial leads.

arrhythmic drugs, cardiac catheterization and even placement of an implantable cardioverter defibrillator. $^{3}$

\section{References}

1. Robottom BJ, Weiner WJ. Teaching neuroImages: rest tremor mimicking ventricular tachycardia. Neurology 2010;75:2134.

2. Huang CY, Shan DE, Lai CH, et al. An accurate electrocardiographic algorithm for differentiation of tremor-induced pseudoventricular tachycardia and true ventricular tachycardia. Int $J$ Cardiol 2006;111:163-5.

3. Knight BP, Pelosi F, Michaud GF, et al. Clinical consequences of electrocardiographic artifact mimicking ventricular tachycardia. N Engl J Med 1999;341:1270-4.

Clinical images are chosen because they are particularly intriguing, classic or dramatic. Submissions of clear, appropriately labelled highresolution images must be accompanied by a figure caption and the patient's written consent for publication. A brief explanation (250 words maximum) of the educational significance of the images with minimal references is required.
Competing interests: None declared.

This article has been peer reviewed.

The authors have obtained patient consent.

Affiliations: Department of Internal Medicine (Martens); Department of Cardiology and Cardiovascular

Medicine (Sinnaeve) University Hospitals Leuven, Gasthuisberg,

Correspondence to:

Pieter Martens,

Pieter.1.martens

@uzleuven.be

CMAJ 2015. DOI:10.1503 /cmaj.140768 Leuven, Belgium 